\title{
Non-Canonical Perturbation Theory of Non-Linear Sigma Models
}

\author{
V. Aldaya ${ }^{1}$, M. Calixto ${ }^{2,1 *}$ and F.F. López-Ruiz ${ }^{1}$ \\ ${ }^{1}$ Instituto de Astrofísica de Andalucía (IAA-CSIC), Apartado Postal 3004, 18080 Granada, \\ Spain \\ 2 Departamento de Matemática Aplicada y Estadística, Universidad Politécnica de Cartagena, \\ Paseo Alfonso XIII 56, 30203 Cartagena, Spain
}

\begin{abstract}
We explore the $O(N)$-invariant Non-Linear Sigma Model (NLSM) in a different perturbative regime from the usual relativistic-free-field one, by using non-canonical basic commutation relations adapted to the underlying $O(N)$ symmetry of the system, which also account for the non-trivial (non-flat) geometry and topology of the target manifold.
\end{abstract}

PACS: 02.20.Tw, 03.70.+k, 11.10.Lm, 11.15.Bt, 12.15.-y

\section{Introduction}

From an abstract (mathematical) point of view, a Non-Linear Sigma Model (NLSM) consists of a set of coupled scalar fields $\pi^{a}\left(x^{\mu}\right), a=1, \ldots, N$, in a $D$-dimensional (Minkowski) spacetime $M$ with coordinates $x^{\mu}, \mu=0,1,2, \ldots, D-1$, and action integral (we use the Einstein summation convention)

$$
S_{\sigma}\left(\pi, \partial_{\mu} \pi\right)=\int_{M} \mathcal{L}\left(\pi, \partial_{\mu} \pi\right) \mathrm{d}^{D} x=\frac{\lambda}{2} \int_{M} g_{a b}(\pi) \partial^{\mu} \pi^{a} \partial_{\mu} \pi^{b} \mathrm{~d}^{D} x,
$$

where $\partial^{\mu}=\eta^{\mu \nu} \partial_{\nu}, \partial_{\nu}=\partial / \partial x^{\nu}, \eta=\operatorname{diag}(+,-, \ldots,-)$ is the Minkowski metric and $\lambda$ a coupling constant. The field theory (11) is called the NLSM with metric $g_{a b}(\pi)$ (usually a positive-definite field-dependent matrix). The fields $\pi^{a}$ themselves could also be considered as the coordinates of an internal Riemannian (target) manifold $\Sigma$ with metric $g_{a b}$. This model proved to be relevant in String Theory where $g_{a b}$ is the Einstein metric and $M$ is a two-dimensional manifold named "worldsheet". An interesting case for us is that in which $\Sigma$ is a Lie group manifold $G$, namely $G=O(N)$, or a quotient (coset) space $G / H$ by a closed subgroup $H$, namely $H=O(N-1)$ (see [3] for $G=U(N)$ and its cosets $G / H$ : complex projective, Grassmann and flag manifolds).

Apart from String Theory, the NLSM is related to a great number of physical systems (see e.g. [1] for a review). It was originally introduced to describe pion dynamics in the theory of strong nuclear interactions. Also, some particular two-dimensional $O(N)$-invariant NLSM are used in connection to antiferromagnetic spin chains, quantum Hall effect and superfluid helium-3. At

${ }^{*}$ Corresponding author: Manuel.Calixto@upct.es 
a more fundamental level, NLSM describes the dynamics of Goldstone bosons in spontaneously broken field theories like the Standard Model of electro-weak interactions. Recently we have proposed in [2] a Higgs-less mechanism to provide mass to the electro-weak gauge vector bosons $W_{ \pm}$and $Z$ through a coupling to a $U(2)$-invariant NLSM à la Stueckelberg. Actually, according to the widely named "Equivalence Theorem" [5, 6], a very heavy Higgs particle can be eliminated from the broken symmetry programme in favor of non-linear $\sigma$-like Goldstone bosons, so that the actual computation of Feynman diagrams involving the longitudinal polarizations of the (massive) vector bosons in electroweak interactions can be resolved in terms of the corresponding diagrams among those scalar fields. Unfortunately, the use of a NLSM Lagrangian has led to an apparent insoluble dichotomy unitarity-renormalizability [7, 8, 9, 10, 11] (see also the review [12] and references therein). In fact, it is well known that the NLSM, in general, suffers from unavoidable renormalizability problems under the canonical quantization programme (see, for instance, [1]). Canonical perturbation theory proceeds from the action (1) by expanding $g_{a b}(\pi)=\delta_{a b}+O\left(\pi^{2}\right)$ and perturbing around massless fields fulfilling $\partial_{\mu} \partial^{\mu} \pi^{a}=0$. However, this perturbation scheme is subject to criticism. On the one hand, massless solutions do not exhaust the whole solution manifold, as other (soliton, instanton, skyrmion) solutions are known to exist [4]. On the other hand, the non-trivial (non-flat) geometry and topology of the target manifold $\Sigma$ and its possible symmetries are not being taken into account or properly exploited. Regarding the last issue, references like [15] tackled the perturbation theory for NLSM in terms of left-Ginvariant quantities $L_{\mu}(x)=g^{-1}(x) \partial_{\mu} g(x), g \in G$, which do not depend on the parametrization of $G$.

As in Reference [13], we think that the trouble that canonical quantization faces in dealing with systems bearing non-trivial topology can be traced back to the "tangent space" approximation imposed at the very beginning of the (canonical) quantization program. Already in the simple case of "free" particles moving on spheres, a proper quantization requires the replacement of canonical commutators with the Lie-algebra commutators of the Euclidean group [13, 14. We shall pursue this idea in this letter and construct a perturbation theory adapted to non-canonical (namely, Euclidean) commutation relations for the particular case of $G=O(N+1)$ invariant NLSM with $\Sigma=S^{N}=O(N+1) / O(N)$ the $N$-dimensional sphere. The discretization of the corresponding equations of motion provides a mechanical picture of the $O(N)$-invariant NLSM as a $(D-1)$-dimensional lattice model of coupled rotators connected by springs (see later on Sec. 2). Actually, this equivalence has already been considered in, for instance, [16, 17] who used the so-called "coupled cluster method" to approach this problem. Our aim here is to explore the NLSM in a different regime from the usual (relativistic-free-field) one, by using non-canonical basic commutation relations adapted to the underlying $O(N)$ symmetry of the system.

\section{$2 O(N+1)$-Invariant NLSM}

The $O(N+1)$-invariant NLSM Lagrangian in (11) can be obtained from the quadratic one

$$
\mathcal{L}\left(\vec{\phi}, \partial_{\mu} \vec{\phi}\right)=\frac{1}{2} \partial_{\mu} \vec{\phi} \cdot \partial^{\mu} \vec{\phi}, \quad \vec{\phi}=\left(\phi^{1}, \ldots, \phi^{N+1}\right) \in \mathbb{R}^{N+1}
$$

with the constraint $\vec{\phi}^{2}=\rho^{2}=$ constant. A NLSM action of type (1) can be recovered from this Lagrangian by eliminating $\phi^{N+1}$ in terms of $\vec{\pi}=\left(\phi^{1}, \ldots, \phi^{N}\right)$ or its stereographic projection 
on $\mathbb{R}^{N}$. Here we shall work with $\vec{\phi}$ and keep in mind the constraint $\vec{\phi}^{2}=\rho^{2}$. Using Lagrange multipliers, the Euler-Lagrange equations of motion can be cast in the form:

$$
\square \vec{\phi}=\frac{\square \vec{\phi} \cdot \vec{\phi}}{\vec{\phi}^{2}} \vec{\phi}, \quad \vec{\phi}^{2}=\rho^{2}
$$

where $\square=\partial_{\mu} \partial^{\mu}$ denotes the d'Alembertian or wave operator. For $N=3, D=2$, extra WessZumino-Novikov-Witten terms can be added to the Lagrangian (2) so that the model is known to be integrable since one is able to find an infinite number of conserved quantities closing a Kac-Moody Lie algebra (see e.g. [1]).

Let us briefly remind how the NLSM above also arises from a $\phi^{4}$-theory by "freezing out" the Higgs field degree of freedom (as in the above-mentioned Equivalence Theorem). Actually, the term "sigma" makes reference to the original model for an effective theory of the meson part of the low-energy nuclear theory. The Lagrangian (2) is modified by a Higgs potential

$$
\mathcal{L}_{g}=\frac{1}{2} \partial_{\mu} \vec{\phi} \cdot \partial^{\mu} \vec{\phi}+\frac{g}{4}\left(\vec{\phi}^{2}-\rho^{2}\right)^{2},
$$

with $g$ a positive constant. It is customary to write

$$
\phi^{N+1}=\rho+\sigma, \phi^{a}=\pi^{a}, a=1, \ldots, N,
$$

for small perturbations around $\vec{\phi}_{(0)}=(0, \ldots, \rho)$. The Lagrangian (4) acquires then the following form in terms of $(\vec{\pi}, \sigma)$ :

$$
\mathcal{L}_{g}=\frac{1}{2} \partial_{\mu} \vec{\pi} \cdot \partial^{\mu} \vec{\pi}+\frac{1}{2} \partial_{\mu} \sigma \partial^{\mu} \sigma+\frac{m_{\sigma}^{2} c^{2}}{2} \sigma^{2}+\ldots
$$

which states that the $\sigma$-meson (Higgs field) has mass $m_{\sigma}=\sqrt{2 g} \rho / c$ whereas the $\pi$-mesons (pions) remain massless. In fact, in the quantum theory, $\pi^{a}$ describe Goldstone bosons associated with the spontaneous breakdown from the $O(N+1)$ to the $O(N)$ symmetry for the choice of vacuum $\left\langle 0\left|\phi^{j}\right| 0\right\rangle=\rho \delta_{j, N+1}$.

The original NLSM Lagrangian (2) can be obtained from (44) by taking the limit $g \rightarrow \infty$ and imposing $\vec{\phi}^{2}=\rho^{2}$ in order to keep the Lagrangian finite except for an irrelevant c-number term. This corresponds to $m_{\sigma} \rightarrow \infty$ so that the Higgs field degree of freedom has been frozen (something physically reasonable since it has not been experimentally observed yet). Note that, even for large $g$, we could always keep $m_{\sigma}$ finite by taking the vacuum expectation value $\rho$ small. Actually, we are interested in this regime in this article.

However, one should be very cautious in taking this limit, since we are dramatically changing the topology of the field configuration space. One can not guarantee in principle that the procedure of perturbing commutes with that of constraining. In this article we pursue the alternative strategy of "constraining and then perturbing", instead of the previous scheme of "perturbing and then constraining". Nowadays it is widely known that constraining does not actually commute (in general) with quantizing (see e.g. [18, 19, 20, 21] for discussions on nonequivalent quantizations of systems with non-trivial configuration spaces).

Let us restrict ourselves, for the sake of simplicity, to the $N=2$ case. The equations (3) can also be obtained as Hamiltonian equations of motion

$$
\dot{\vec{\phi}}=\frac{\partial \vec{\phi}}{\partial t} \equiv\{\vec{\phi}, H\}, \ddot{\vec{\phi}}=\frac{\partial^{2} \vec{\phi}}{\partial t^{2}} \equiv\{\dot{\vec{\phi}}, H\}=\{\{\vec{\phi}, H\}, H\},
$$


for the Hamiltonian

$$
H=\frac{1}{2} \int d^{D-1} x\left(\frac{\vec{L}^{2}(x)}{\rho^{2}}+c^{2}(\vec{\nabla} \vec{\phi}(x))^{2}\right),
$$

and the basic equal-time Euclidean (non-canonical) Poisson brackets

$$
\left\{L^{i}(x), L^{j}(y)\right\}=\epsilon_{k}^{i j} L^{k}(x) \delta(x-y), \quad\left\{L^{i}(x), \phi^{j}(y)\right\}=\epsilon_{k}^{i j} \phi^{k}(x) \delta(x-y),
$$

where $\vec{L} \equiv \vec{\phi} \wedge \dot{\vec{\phi}},(\vec{\nabla} \vec{\phi})^{2} \equiv \partial_{j} \phi_{k} \partial^{j} \phi^{k}, \epsilon^{i j}{ }_{k}$ is the antisymmetric symbol and we have introduced the wave velocity $c$ when setting $x^{0}=c t$ for later convenience. Actually, if (9) are taken as abstract Poisson brackets, with $\vec{L}$ not necessarily related to $\vec{\phi}$, then the equations (7) generalize (3) by introducing an extra term

$$
\square \vec{\phi}=\frac{\square \vec{\phi} \cdot \vec{\phi}}{\vec{\phi}^{2}} \vec{\phi}+\frac{\vec{L} \cdot \vec{\phi}}{\vec{\phi}^{4}} \vec{L}
$$

which could not vanish when $\vec{L} \cdot \vec{\phi} \neq 0$, a situation which arises when "magnetic monopoles" are present and $\vec{L}$ is not necessarily perpendicular to $\vec{\phi}$. We shall restrict ourselves to the case $C_{1}=\vec{L} \cdot \vec{\phi}=0$, which is compatible with the Poisson brackets (9) and the constraint $C_{2}=\vec{\phi}^{2}=\rho^{2}$, since both $C_{1}$ and $C_{2}$ are the natural Casimir operators for the Euclidean group.

Perturbing around $\vec{\phi}_{(0)}=(0,0, \rho)$ as in (5) , for fixed $\vec{\phi}^{2}=\rho^{2}$, can be interpreted as a "group contraction", which drastically changes the topology of the system. Indeed, this perturbation theory has sense for $\rho \gg 1$. Making the change (5) in the last Poisson bracket of (9) and taking the limit $\rho \rightarrow \infty$, keeping $\varphi^{1,2} \equiv \pi^{1,2} / \rho$ finite, we recover the canonical Poisson brackets:

$$
\left\{L^{i}(x), \varphi^{j}(y)\right\}=\epsilon^{i j}{ }_{3} \delta(x-y), \quad i, j=1,2
$$

which state that $\left(\varphi^{1}, \varphi^{2}\right)$ and $\left(L^{2},-L^{1}\right)$ are couples of canonically-conjugated variables. Therefore, standard (canonical) perturbation theory has sense for large values of $\rho$, which loses information about the (compact) topology of the system. As already commented, we are interested in the other regime $\rho \ll 1$.

\section{Classical non-canonical perturbation theory}

A solution of $\square \vec{\phi}=-m^{2} \vec{\phi}$, for any constant $m$, is also a solution of (3). However, only for massless fields, $m=0$, the constraint $\vec{\phi}^{2}=\rho^{2}$ is also satisfied. At least at the quantum level, standard perturbation theory proceeds by considering scattering of massless fields $\vec{\phi}[1]$. However, at the classical level, we know that there are more solutions of (3) than massless solutions. In fact, as showed long time ago in [4, the configuration space of a NLSM breaks up into an (infinite) number of components. Indeed, finite energy requires boundary conditions like (for instance) $\vec{\phi}(x)=(0,0, \rho)$ as $\|x\| \rightarrow \infty$, which means a one-point compactification of $\mathbb{R}^{D-1}$ by $S^{D-1}$. Thus, if two fields $\vec{\phi}$ and $\vec{\phi}^{\prime}$ belong to different homotopical classes $\Pi_{D-1}\left(S^{N}\right)$, then they can not be continuously deformed (evolved) one into the other. In particular, one can find (solitonic) solutions that are not wave packets of massless solutions. 
Instead of perturbing around massless solutions, we shall adopt the following splitting of the Hamiltonian (8)

$$
H=H_{0}+V, \quad H_{0}=\frac{1}{2} \int d^{D-1} x \frac{\vec{L}^{2}(x)}{\rho^{2}}, \quad V=\frac{c^{2}}{2} \int d^{D-1} x(\vec{\nabla} \vec{\phi}(x))^{2},
$$

and consider $V$ as a perturbation for either small $c$ or $\|\vec{\phi}\|=\rho \ll 1$ (with $c$ arbitrary).

Given an initial condition on a Cauchy hypersurface, $\vec{\phi}\left(t_{0}, x\right)=\vec{\phi}_{0}(x)$ and $\dot{\vec{\phi}}\left(t_{0}, x\right)=\dot{\vec{\phi}}_{0}(x)$, the general solution to (3) can be formally written as:

$$
\vec{\phi}(t, x)=e^{\left(t-t_{0}\right)\{\cdot, H\}} \vec{\phi}_{0}(x)=U\left(t-t_{0}\right) \vec{\phi}_{0}(x),
$$

where $\{\cdot, H\}$ stands for the Liouvillian operator and $U\left(t-t_{0}\right)=e^{\left(t-t_{0}\right)\{\cdot, H\}}$ for the evolution operator. Actually, we can exactly integrate the "free" evolution as:

$$
\begin{aligned}
\vec{\phi}^{(0)}(t, x) & \equiv e^{\left(t-t_{0}\right)\left\{\cdot, H_{0}\right\}} \vec{\phi}_{0}(x)=U_{0}\left(t-t_{0}\right) \vec{\phi}_{0}(x) \\
& =\cos \left(\sqrt{\frac{\vec{L}^{2}(x)}{\rho^{4}}}\left(t-t_{0}\right)\right) \vec{\phi}_{0}(x)+\frac{1}{\sqrt{\frac{\vec{L}^{2}(x)}{\rho^{4}}}} \sin \left(\sqrt{\frac{\vec{L}^{2}(x)}{\rho^{4}}}\left(t-t_{0}\right)\right) \dot{\vec{\phi}}_{0}(x) .
\end{aligned}
$$

We shall let the wave velocity $c$ to take arbitrary values, as we want our perturbation theory to be valid for relativistic fields too. We have already justified the interesting regime $\rho \ll 1$ (small vacuum expectation value) in which the Higgs mass $m_{\sigma}$ would remain finite while $\vec{\phi}^{2} \simeq \rho^{2}$, so that the Higgs field degree of freedom is almost frozen. In order to gain more physical intuition on this limit, let us use the following mechanical picture of coupled small rotators (see Figure 11).

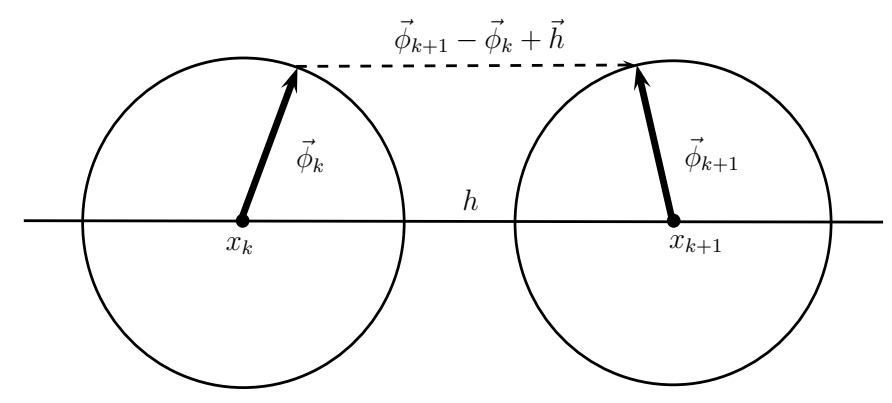

Figure 1: Rotators in a lattice coupled by springs

Without loss of generality, we can restrict ourselves to $D=2$, consider the lattice $x_{k}=$ $k h, k \in \mathbb{Z}$, for some (small) step $h$, and write $\vec{\phi}_{k}(t)=\vec{\phi}\left(t, x_{k}\right)$ for the vector position of the rotator in the place $x_{k}$. Rotators are connected by identical springs of constant $\kappa$ and zero natural length so that the elastic potential energy between two consecutive rotators is

$$
V_{k+1, k}=\frac{1}{2} \kappa\left(\vec{\phi}_{k+1}-\vec{\phi}_{k}+h(1,0)\right)^{2} .
$$


Taking the limit $h \rightarrow 0$, keeping $\kappa h \equiv c^{2}$ finite, we have that the total elastic potential energy is

$$
\sum_{k=-\infty}^{\infty} V_{k+1, k}=\frac{1}{2} c^{2} \sum_{k=-\infty}^{\infty} h \frac{\left(\vec{\phi}_{k+1}-\vec{\phi}_{k}+h(1,0)\right)^{2}}{h^{2}} \rightarrow \frac{1}{2} c^{2} \int_{-\infty}^{\infty} d x\left(\left(\partial_{x} \vec{\phi}\right)^{2}+2 \partial_{x} \phi^{1}\right) .
$$

which gives the desired result up to a boundary term.

Contrary to the (unconstrained) Klein-Gordon field (as a model of coupled oscillators), the elastic potential energy $V$ can be made arbitrarily small for NLSM fields (as a model of coupled rotators) by taking $\rho \ll 1$, even in rigid media ( $c$ arbitrary). In other words, unlike a NLSM, a Klein-Gordon field could never be seen as an infinite set of weakly coupled oscillators unless inside soft media $(c \ll 1)$ where it takes a long time for the wave to propagate. That is, here we have the vacuum expectation value $\rho$ as an extra perturbation parameter to play with.

Although Dyson series are conventionally designed for quantum perturbation theory, we shall briefly remind the subject here in a classical setting. Dyson series takes advantage of the exact solvability of $H_{0}$, with exact solution (14), to provide a perturbation series in $V$. The evolution operator (13) is decomposed as:

$$
U\left(t, t_{0}\right)=U_{0}(t) \underbrace{U_{0}(-t) U\left(t-t_{0}\right) U_{0}\left(t_{0}\right)}_{U_{I}\left(t, t_{0}\right)} U_{0}\left(-t_{0}\right),
$$

where $U_{I}\left(t, t_{0}\right)$ is the evolution operator in the interaction image. Let us set $t_{0}=0$ for simplicity. After a little bit of algebra, one can see that

$$
\frac{\partial}{\partial t} U_{I}(t)=\hat{V}(t) U_{I}(t), \quad \hat{V}(t) \equiv U_{0}(t)\{\cdot, V(\vec{\phi})\} U_{0}(-t)=\left\{\cdot V\left(\vec{\phi}^{(0)}(-t)\right)\right\},
$$

with $\vec{\phi}^{(0)}(t)$ given by (14) (note the time inversion). This formula can be recursively integrated as:

$$
U_{I}(t)=I+\int_{0}^{t} d \tau \hat{V}(\tau)+\int_{0}^{t} d \tau \int_{0}^{\tau} d \tau^{\prime} \hat{V}(\tau) \hat{V}\left(\tau^{\prime}\right)+\ldots
$$

In order to test the perturbation procedure, let us consider the exactly solvable case $N=1, D=$ 2. On the one hand, if we parametrize the field $\vec{\phi}=\left(\phi^{1}, \phi^{2}\right)$ in polar coordinates $\phi=\rho e^{i \theta}$, then (3) reduces to a massless Klein-Gordon equation for $\square \theta=0$. On the other hand, we can compute order by order:

$$
\phi(t, x)=U(t) \phi_{0}(x)=U_{0}(t) U_{I}(t) \phi_{0}(x)=U_{0}(t) \phi^{(I)}(t, x),
$$

with

$$
\phi^{(I)}(t, x)=U_{I}(t) \phi_{0}(x)=\phi_{0}(x)+\int_{0}^{t} d \tau\left\{\phi_{0}(x), V\left(\phi^{(0)}(-\tau)\right)\right\}+\ldots,
$$

where $V(\phi)=\frac{c^{2}}{2} \int_{-\infty}^{\infty} d x \partial_{x} \phi \partial_{x} \bar{\phi}$ and Poisson brackets are computed at $\tau=0$. Taking into account that

$$
\phi^{(0)}(\tau, x)=U_{0}(\tau) \phi_{0}(x)=e^{i \tau L(x) / \rho^{2}} \phi_{0}(x)
$$

with $L=\phi^{1} \dot{\phi}^{2}-\phi^{2} \dot{\phi}^{1}=\operatorname{Im}(\bar{\phi} \dot{\phi})$, and that

$$
\{L(x), \phi(y)\}=-i \phi(x) \delta(x-y),\{\Psi(L(x)), \phi(y)\}=\Psi^{\prime}(L(x))\{L(x), \phi(y)\},
$$


for any derivable function $\Psi$ of $L$, we can compute at first order:

$$
\begin{aligned}
\phi_{0}^{(1)}(\tau, x) & \equiv\left\{\phi_{0}(x), V\left(\phi^{(0)}(-\tau)\right)\right\} \\
& =\frac{c^{2}}{2}\left(\partial_{x x}^{2} \phi^{(0)}(-\tau, x) \tau e^{i \tau L(x) / \rho^{2}}-\partial_{x x}^{2} \bar{\phi}^{(0)}(-\tau, x) \frac{\tau}{\rho^{2}} e^{-i \tau L(x) / \rho^{2}} \phi_{0}^{2}(x)\right) \\
& =\frac{i c^{2} \tau}{\rho^{2}}\left(\rho^{2} \partial_{x x}^{2} \theta_{0}(x)-\tau \partial_{x x}^{2} L(x)\right) \phi_{0}(x),
\end{aligned}
$$

where we have put $\phi_{0}(x)=\rho e^{i \theta_{0}(x)}$. Therefore,

$$
\begin{aligned}
\phi^{(I)}(t, x) & =U_{I}(t) \phi_{0}(x)=\phi_{0}(x)+\int_{0}^{t} d \tau \phi_{0}^{(1)}(\tau, x)+\ldots \\
& =\phi_{0}(x)\left(1+\frac{i c^{2} t^{2}}{2 \rho^{2}}\left(\rho^{2} \partial_{x x}^{2} \theta_{0}(x)-\frac{2}{3} t \partial_{x x}^{2} L(x)\right)+\ldots\right) .
\end{aligned}
$$

The last step in (20), i.e. $\phi(t)=U_{0}(t) \phi^{(I)}(t)$, is easily performed by replacing $\phi_{0}(x)$ by $\phi^{(0)}(t, x)$ (and $\theta_{0}(x)$ by $\theta^{(0)}(t, x)=\theta_{0}(x)+t L(x) / \rho^{2}$ ) everywhere in $\phi^{(I)}(t, x)$. That is:

$$
\phi(t, x)=U_{0}(t) \phi^{(I)}(t, x)=\phi^{(0)}(t, x)\left(1+\frac{i c^{2} t^{2}}{2}\left(\partial_{x x}^{2} \theta_{0}(x)+\frac{1}{3 \rho^{2}} t \partial_{x x}^{2} L(x)\right)+\ldots\right) .
$$

One can check that, at this order, the perturbative solution coincides with the exact solution $\phi(t, x)=\rho e^{i \theta(t, x)}$ where

$$
\theta(t, x)=\cos \left(c t \partial_{x}\right) \theta_{0}(x)+\frac{\sin \left(c t \partial_{x}\right)}{c \partial_{x}} \dot{\theta}_{0}(x)
$$

Inside the discrete, mechanical picture depicted in Figure 1, the appearance of second order spatial derivatives $\partial_{x x}^{2}$ at first order in perturbation theory means that the interaction propagates from one point $x_{k}$ to its nearest neighbors $x_{k+1}$ and $x_{k-1}$ at this order. In order to account for a longer range propagation we should go to higher orders in perturbation theory.

\section{Quantum non-canonical perturbation theory}

In quantum field theory the fields $\phi(x)$ and $L(x)$ are promoted to the quantum operators $\hat{\phi}$ and $\hat{L}$, respectively, and the Poisson brackets (9) and (23) are promoted to the (non-canonical) commutators (we shall keep restricting ourselves to $N=1, D=2$, for simplicity):

$$
[\hat{L}(x), \hat{\phi}(y)]=\hbar \hat{\phi}(x) \delta(x-y), \quad\left[\hat{L}(x), \hat{\phi}^{\dagger}(y)\right]=-\hbar \hat{\phi}^{\dagger}(x) \delta(x-y),
$$

where we have introduced $\hbar$ just to account for quantum corrections and $\rho^{2}=\hat{\phi}(x) \hat{\phi}^{\dagger}(x)$ gets the necessary dimensions to render the Hamiltonian with energy dimensions. Let us consider the lattice picture of our field model and write $\hat{\phi}\left(x_{k}\right)=\hat{\phi}_{k}$ and $\hat{L}\left(x_{k}\right)=\hat{L}_{k}$. The Hilbert space $\mathcal{H}_{k}=\operatorname{Span}\left(\left|n_{k}\right\rangle, n_{k} \in \mathbb{Z}\right)$ of a single rotator at position $x_{k}$ is spanned by the (normalized) eigenstates $\left|n_{k}\right\rangle$ of the angular momentum $\hat{L}_{k}$, that is:

$$
\hat{L}_{k}\left|n_{k}\right\rangle=\hbar n_{k}\left|n_{k}\right\rangle \text {. }
$$


The operators $\hat{\phi}_{k}$ and $\hat{\phi}_{k}^{\dagger}$ act on $\left|n_{k}\right\rangle$ as ladder operators, namely:

$$
\hat{\phi}_{k}\left|n_{k}\right\rangle=\rho\left|n_{k}+1\right\rangle, \quad \hat{\phi}_{k}^{\dagger}\left|n_{k}\right\rangle=\rho\left|n_{k}-1\right\rangle .
$$

The total Hilbert space $\mathcal{H}$ of our lattice quantum field theory will be the direct product $\mathcal{H}=$ $\bigotimes_{k \in \mathbb{Z}} \mathcal{H}_{k}$. The total Hamiltonian operator is

$$
\hat{H}=\hat{H}_{0}+\hat{V}, \quad \hat{H}_{0}=\frac{\omega}{2 \hbar} \sum_{k=-\infty}^{\infty} \hat{L}_{k}^{2}, \quad \hat{V}(\hat{\phi})=-\kappa \sum_{k=-\infty}^{\infty} \operatorname{Re}\left(\hat{\phi}_{k+1} \hat{\phi}_{k}^{\dagger}\right)
$$

where we have discarded a c-number addend in $\hat{V}$ and we have introduced a frequency $\omega \equiv h \hbar / \rho^{2}$. In order to write the evolution operator in the interaction image $U_{I}(t)$, we need to evolve $\phi_{k}$ with the free evolution operator $U_{0}(t)=e^{-\frac{i t}{\hbar} \hat{H}_{0}}$ :

$$
\hat{\phi}_{k}^{(0)}(t)=U_{0}(-t) \hat{\phi}_{k} U_{0}(t)=\sum_{m=0}^{\infty} \frac{(-i t / \hbar)^{m}}{m !}\left[\hat{\phi}_{k}, \hat{H}_{0}\right]^{(m)},
$$

where we denote the multiple commutator:

$$
\left[\hat{\phi}_{k}, \hat{H}_{0}\right]^{(m)} \equiv\left[\left[\hat{\phi}_{k}, \hat{H}_{0}\right], \stackrel{m}{.}, \hat{H}_{0}\right]
$$

The quantum commutator introduces new ordering problems with respect to the classical Poisson bracket. For standard creation $\hat{a}_{k}^{\dagger}$ and annihilation $\hat{a}_{k}$ operators, Wick's theorem provides a useful tool for writing arbitrary products of $\hat{a}_{k}^{\dagger}$ and $\hat{a}_{l}$ in terms of normal ordered products. Here we have to deduce a new Wick-like theorem in order to write arbitrary products of the non-canonical operators $\hat{L}_{k}$ and $\hat{\phi}_{l}$. If we choose by convention to write all $\hat{L}$ 's to the left of all $\hat{\phi}$ 's, then the multiple commutator (34) acquires the following form:

$$
\left[\hat{\phi}_{k}, \hat{H}_{0}\right]^{(m)}=\frac{(-1)^{m} \omega^{m}}{2^{m}}\left(\sum_{l=0}^{m} c_{m, l} \hbar^{l} \hat{L}_{k}^{m-l}\right) \hat{\phi}_{k}=(-1)^{m} \omega^{m}\left(\hat{L}_{k}^{m}+q . c .\right) \hat{\phi}_{k}
$$

where q.c. stands for "quantum corrections". The Wick-like numerical coefficients $c_{m, l}$ are given by $c_{m, 0}=2^{m}, c_{m, m}=(-1)^{m}$ and the recurrence $c_{m, l}=2 c_{m-1, l}-c_{m-1, l-1}$. Therefore

$$
\hat{\phi}_{k}^{(0)}(t)=U_{0}(-t) \hat{\phi}_{k} U_{0}(t)=\left(e^{i t \omega \hat{L}_{k} / \hbar}+q . c .\right) \hat{\phi}_{k},
$$

coincides with the classical expression (22) except for quantum corrections. Actually, we shall be able to sum up all quantum corrections in some particular cases (see later) by noticing that

$$
\sum_{l=0}^{m} c_{m, l}=1, \quad \forall m=0,1,2, \ldots
$$

The evolution operator in the interaction image (19) is given in terms of

$$
\hat{V}\left(\hat{\phi}^{(0)}(-\tau)\right)=-\kappa \sum_{q=-\infty}^{\infty} \operatorname{Re}\left(\left(e^{-i \tau \omega \hat{L}_{q+1} / \hbar}+q . c .\right) \hat{\phi}_{q+1} \hat{\phi}_{q}^{\dagger}\left(e^{i \tau \omega \hat{L}_{q} / \hbar}+q . c .\right)\right) .
$$


In order to describe the new perturbation scheme, let us consider an initial state (at time $t=0$ )

$$
|\{n\}\rangle=\otimes_{q \in \mathbb{Z}}\left|n_{q}\right\rangle .
$$

The probability amplitude of observing $\left|\left\{n^{\prime}\right\}\right\rangle$ as a final state after time $t$ is given by the $S$ matrix element:

$$
S_{n, n^{\prime}}(t)=\left\langle\left\{n^{\prime}\right\}|U(t)|\{n\}\right\rangle=\left\langle\left\{n^{\prime}\right\}\left|U_{0}(t) U_{I}(t)\right|\{n\}\right\rangle=e^{i t \frac{\omega}{2} \sum_{k=-\infty}^{\infty}\left(n_{k}^{\prime}\right)^{2}}\left\langle\left\{n^{\prime}\right\}\left|U_{I}(t)\right|\{n\}\right\rangle .
$$

The total angular momentum $\hat{L}=\sum_{k} \hat{L}_{k}$ is conserved at all orders in perturbation theory since $[\hat{L}, \hat{V}]=0$. This means that

$$
\sum_{k=-\infty}^{\infty} n_{k} \neq \sum_{k=-\infty}^{\infty} n_{k}^{\prime} \Rightarrow S_{n, n^{\prime}}(t)=0
$$

The interaction potential (38) is of short range, that is, $\hat{V}$ is not able to carry one quantum of angular momentum from position $k$ to $l$ until $|k-l|$-th order in perturbation theory. More precisely, considering an initial state of the form

$$
\left|\left\{\delta_{k}\right\}\right\rangle=\otimes_{q \in \mathbb{Z}}\left|\delta_{k, q}\right\rangle
$$

we can compute the probability amplitude of observing $\left|\left\{\delta_{l}\right\}\right\rangle$ as a final state after time $t$ at all orders:

$$
S_{\delta_{k}, \delta_{l}}(t)=e^{i t \omega / 2} \sum_{n=0}^{\infty}\left(\frac{i t \kappa \rho^{2}}{2 \hbar}\right)^{n} \frac{1}{n !} \sum_{s=0}^{n}\left(\begin{array}{l}
n \\
s
\end{array}\right) \delta_{l, k-n+2 s}
$$

where we have made use of (37) at some stage. Note that perturbation theory is dictated by both: $\kappa$ and/or $\rho$.

Instead of the angular momentum eigenstates $\left|n_{k}\right\rangle$ we could also have used field eigenstates

$$
\left|\zeta_{k}\right\rangle \equiv \sum_{n=-\infty}^{\infty} \zeta_{k}^{n}|n\rangle, \quad\left|\zeta_{k}\right|=1
$$

for which $\hat{\phi}_{k}\left|\zeta_{k}\right\rangle=\rho \zeta_{k}\left|\zeta_{k}\right\rangle, \hat{\phi}_{k}^{\dagger}\left|\zeta_{k}\right\rangle=\rho \zeta_{k}^{-1}\left|\zeta_{k}\right\rangle$ and $\hat{L}_{k}\left|\zeta_{k}\right\rangle=\hbar \zeta_{k} \partial_{\zeta_{k}}\left|\zeta_{k}\right\rangle$. Moreover, going from $N=1$ to arbitrary $N$ can be accomplished by replacing $\left|n_{k}\right\rangle$ with hyper-spherical harmonics. For $N=2$, the usual spherical harmonics are given in terms of homogeneous polynomials of degree $j$ in $\vec{\phi}$ :

$$
Y_{m}^{j}\left(\vec{\phi}_{k}\right)=\sum_{\substack{a_{q}=1,2,3 \\ q=1, \ldots, j}} \xi_{a_{1}, \ldots, a_{j}}^{(m)} \phi_{k}^{a_{1}} \ldots \phi_{k}^{a_{j}},
$$

where $\xi_{a_{1}, \ldots, a_{j}}^{(m)}$ are the complex components of a symmetric and traceless tensor [22]. The angular momentum operator at place $x_{k}$ is then given by $\hat{L}_{k}^{a}=\hbar \epsilon_{c}^{a b} \phi_{k}^{c} \partial_{\phi_{k}^{b}}$, as usual. 


\section{Conclusions}

The usual perturbation theory for relativistic fields is designed for small deviations from the free (Klein-Gordon or Dirac) fields. The data analysis of detectors in particle colliders is also intended for this purpose. However, fields of NLSM-type can be found in a strongly-interacting regime $(\rho \ll 1)$ which does not fit into this picture. This leads us to reconsider the perturbation theory and renormalizability of the NLSM.

In this paper we have considered a non-canonical approach to the perturbation theory of the $O(N)$-invariant NLSM which accounts for the non-trivial (non-flat) geometry and topology of the target manifold $\Sigma$ and takes advantage of the underlying symmetries of the system. This scheme can also be adapted to other $G$-invariant NLSM.

\section{Acknowledgements}

Work partially supported by the Fundación Séneca (08814/PI/08), Spanish MICINN (FIS200806078-C03-01) and Junta de Andalucía (FQM219, FQM1951). F.F. López-Ruiz thanks C.S.I.C. for an I3P grant. M. Calixto thanks the "Universidad Politécnica de Cartagena" and C.A.R.M. for the award "Intensificación de la Actividad Investigadora 2009-2010".

\section{References}

[1] S.V. Ketov, Quantum Non-linear Sigma-Models, Springer-Verlag Berlin Heidelberg (2000).

[2] V. Aldaya, M. Calixto and F.F. López-Ruiz, A Quantizable Model of Massive Gauge Vector Bosons without Higgs, Mod. Phys. Lett. A24 (2009) 2731-2740

[3] V. Aldaya, M. Calixto, F.F. López-Ruiz and E. Sánchez-Sastre, Coupling Nonlinear SigmaMatter to Yang-Mills Fields: Symmetry Breaking Patterns, Journal of Nonlinear Mathematical Physics 15 (2008) 91-101. arXiv:0809.2077

[4] A.A. Belavin and A.M. Polyakov, Metastable states of two-dimensional isotropic ferromagnets, JETP Lett. 22 (1975) 245-247

[5] A.C. Longhitano, Low-energy impact of a heavy Higgs boson sector, Nucl. Phys. B188 (1981) 118-154

[6] W.A. Bardeen and K. Shizuya, Structure and renormalizability of massive Yang-Mills field theories, Phys. Rev. D18 (1978) 1969-1982

[7] R. Delbourgo and G. Thompson, Massive, unitary, renormalizable Yang-Mills theory without Higgs bosons, Phys. Rev. Lett. 57 (1986) 2610-2612

[8] J. Kubo, Comment on "Massive, Unitary, Renormalizable Yang-Mills Theory without Higgs Bosons", Phys. Rev. Lett. 58 (1987) 2000-2000

[9] P. Kosinski and L. Szymanowski, Comment on "Massive, Unitary, Renormalizable YangMills Theory without Higgs Bosons", Phys. Rev. Lett. 58 (1987) 2001-2001 
[10] R. Delbourgo, S. Twisk and G. Thompson, Massive Yang-Mills Theory: Renormalizability Versus Unitarity, Int. J. Mod. Phys. A3 (1988) 435-449

[11] T. Hurth, Higgs-free Massive Nonabelian Gauge Theories, Helv. Phys. Acta 70 (1997) 406-416

[12] H. Ruegg and M. Ruiz-Altaba, The Stueckelberg Field, Int. J. Mod. Phys. A19 (2004) $3265-3348$.

[13] C.J. Isham, Topological and global aspects of quantum theory, in Relativity, Groups and Topology II, Les Houches Summer School, R. Stora and B. S. DeWitt (Eds.) (1986)

[14] V. Aldaya, M. Calixto, J. Guerrero and F.F. Lopez-Ruiz, Group-quantization of nonlinear sigma models: particle on $S^{2}$ revisited, Rep. Math. Phys. 64 (2009) 49-58.

[15] L. D. Faddeev and A. A. Slavnov, Invariant perturbation theory for nonlinear chiral Lagrangians. Theor. Math. Phys. 8 (1971) 843-850

[16] N. E. Ligterink, N. R. Walet and R. F. Bishop, The Ground State of the Nonlinear Sigma Model $O(4)_{3+1}$, Nuclear Physics B (Proc. Suppl.) 63A-C (1998) 667-669

[17] N. E. Ligterink, N. R. Walet and R. F. Bishop, A Coupled-Cluster Formulation of Hamiltonian Lattice Field Theory: The Nonlinear Sigma Model, Annals of Physics 267 (1998) $97-133$

[18] V. Aldaya, J. Navarro-Salas and A. Ramirez, Algebraic quantization on a group and nonabelian constraints, Commun. Math. Phys. 121 (1989) 541

[19] N.P. Landsman and N. Linden, The geometry of inequivalent quantizations, Nucl. Phys. B365 (1991) 121

[20] D. MacMullan and I. Tsutsui, On the emergence of gauge structures and generalized spin when quantizing on a coset space, Ann. Phys. 237 (1995) 269-321

[21] V. Aldaya, M. Calixto and J. Guerrero, Algebraic Quantization, Good Operators and Fractional Quantum Numbers, Commun. Math. Phys. 178 (1996) 399

[22] L.C. Biedenharn and J.D. Louck, Angular Momentum in Quantum Physics, AddisonWesley, Reading, MA, 1981 